\title{
Robust Contrast Enhancement for Digital Mammography
}

\author{
Norhène GARGOURI BEN \\ AYED \\ University of Sfax, Sfax \\ Engineering School \\ Computers Imaging Electronics \\ and Systems Group(CIELS)
}

\author{
Alima DAMAK MASMOUDI \\ University of Sfax, Sfax \\ Engineering School \\ Computers Imaging Electronics \\ and Systems Group(CIELS)
}

\author{
Dorra SELLAMI \\ MASMOUDI \\ University of Sfax, Sfax \\ Engineering School \\ Computers Imaging Electronics \\ and Systems Group(CIELS)
}

\begin{abstract}
Nowadays, mammography is currently considered as the most efficient way for the detection and diagnosis of breast cancer at early stages. In many case, due to the subtleness of the difference between normal features and cancerous ones and the bad imaging conditions, cancer is not easily detected with visual interpretation. Thus, image-enhancement technology is often used in screaming mammograms. In this paper, we develop a method based on Shock Filter to enhance the contrast of image and help radiologists. In the proposed method, the Shock Filter is applied for preprocessing, in order to improve the contrast, remove the noisy fluctuations and to enhance the edges containing useful information. Experiments show the efficiency of the proposed method.
\end{abstract}

\section{General Terms}

Mammography, Enhancement

\section{Keywords}

Mammogram, Image enhancement, Contrast

enhancement, Shock filters.

\section{INTRODUCTION}

Recently, breast cancer is the second leading cause of women's deaths between the age of 35 and 55, especially in the developed countries; it remains the biggest public health problem. But, the early diagnosis of the breast cancer is a key step for reducing mortality rates [1].

Mammography is an efficient way used by radiologists in the detection and diagnosis of breast cancer. It is reviewed as the one of the most reliable method for early detection of tumors in breast cancer.

However, due to the low-dose of X-ray hardware system, mammograms may present modest characteristics; such as the low contrast between normal and malignant tissue, or poor resolution that makes the precise diagnosis very difficult. Therefore, to improve the diagnosis rate of mammogram, many techniques of image enhancement are used for early detection of cancer.

Computer-assisted analysis of mammograms depends on regions of interest (ROI). Hence, the mammographic image enhancement may be expected to preserve the mammogram quality and highlight the features of the image. The nonlinear filters have been used for the enhancement of the tumor areas in the mammogram, among the non-linear filters, Yoshida.H and al. [2] used median filters. The region of support was investigated in the detection and the enhancement. The median filter adds noise into the transformed mammogram. In the presence of the noise, which depends on the shape of the transform window and noise levels, median filter may generate streaks and amorphous blocks. In order to overcome the above limitations, we investigate the mammographic image enhancement technology based on Shock Filter for radiologists to display them clearly with the computer screen. The Shock Filter is applied to remove noisy fluctuations, enhance useful edges and contrast. Knowing that shape, contrast and boundary of region presenting abnormalities are found very important features for tumor detection. That's why the Shock Filter could be useful in performing feature extraction and classification of malignant and benign tumor.

This paper is organized as follows. In Section 2, we present brief survey on existing methods of enhancement. Section 3, presents basic theory of Shock Filter. In Section 4, we present enhancement performance measure and experimental results. In Section 5, conclusion and future work are discussed.

\section{BRIEF SURVEY ON EXISTING METHODS OF ENHANCEMENT}

Contrast enhancement is a method used to determine the optimal transformation function joining the initial gray level with the present intensity. So, contrast between adjacent structures in an image is maximally portrayed [3]. Pisano and al. [4], presented a review of the habitual methods of contrast enhancement corresponding to digital radiography.

\subsection{Contourlet Transform (CT)}

Contourlet Transform was introduced in 2002 [5] by Do and Vetterli as multidirectional and multiscale transform. Other different methods, such as Ridgelet and Curvelet, that first expand a transform in the continuous domain followed by discretization leading to sampled data. In [5], the multidirectional and multiscale transform were constructed in the discrete domain by combining the Laplacian Pyramid (LP) and the directional filter bank using non-separable filter banks, as shown in Figure 1. The LP is used at the beginning to capture the point discontinuities, and then followed by a directional filter bank (DFB) to link discontinuous points into linear structures. 


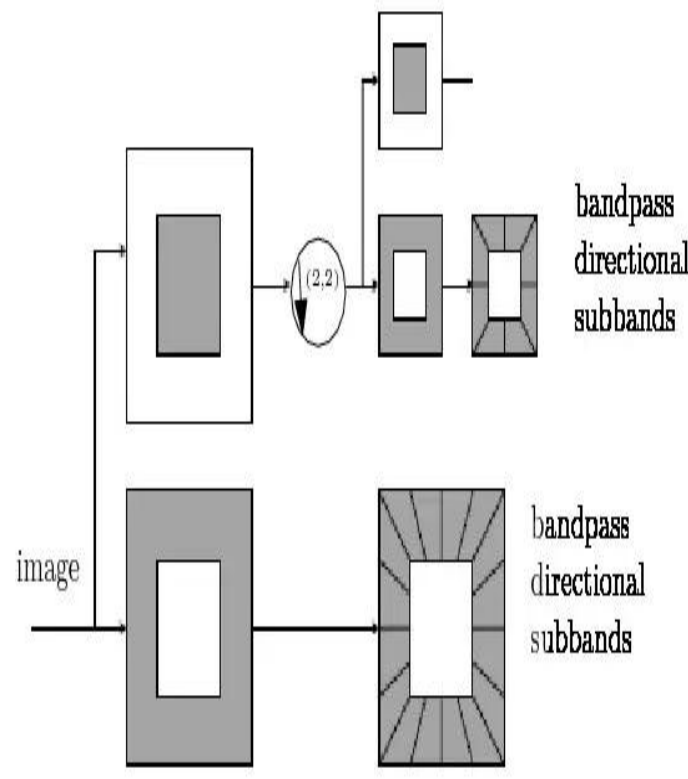

Fig 1: Processing steps of Contourlet transform

In [6], Cunha et al. developed the Nonsubsampled Contourlet Transform (NSCT).

Figure 2 displays an outline of the proposed NSCT. The proposed NSCT is a fully shift-invariant, multiscale, and multidirection version of the Contourlet Transform. The NSCT is composed of two steps:

- a nonsubsampled pyramid NSP operation that guarantees the multiscale property;

- a nonsubsampled DFB operation that provides directionality.

Due to its shift-invariant property, we can improve results in the image processing tasks; such as image enhancement or denoising.

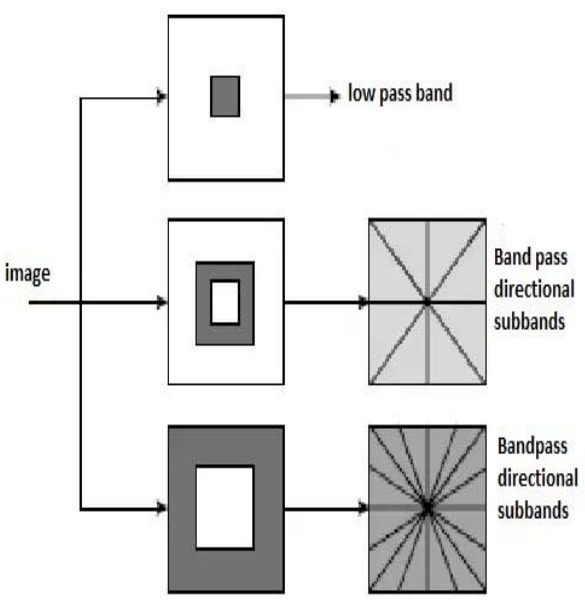

Fig 2: Processing steps of Contourlet Transform

Moreover, it is extra flexible for the design of the filter. Enhancement results of a mammogram using NSCT is represented in figure 3 .

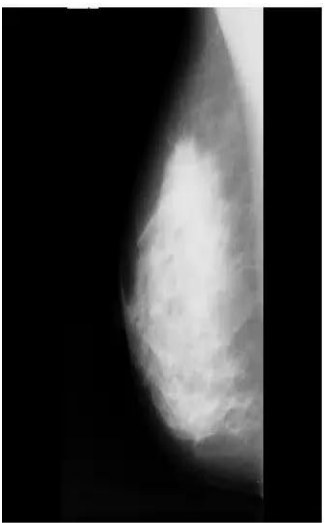

(a)

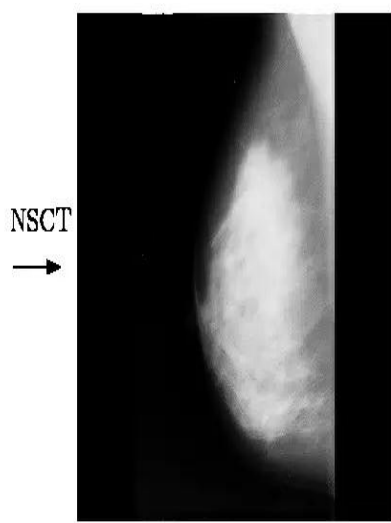

(b)
Fig3: Mammogram enhancement based on NSCT. (a)Original mammogram; (b) Enhanced mammogram

\subsection{Histogram Equalization and CLAHE}

\subsubsection{Histogram Equalization (HE)}

In image processing, $\mathrm{HE}$ is a method adjusting the contrast of a digital image, this technique is developed for image enhancement, it consist on applying a transformation on each pixel of the image, and so on to get a new image from an independent operation of each pixel. This transformation is constructed from the cumulative histogram of the original image.

The HE allows a better distribution of the intensities of the entire range of possible values by spreading histogram.

Adaptive HE (AHE) divides the images into regions and performs local HE. It is a standard technique based on local histogram to get the final pixel gray level value, which provides a local contrast enhancement [7]. The use of standard or adaptative histogram equalization may cause a noise amplification in flat regions within the image with ring artifacts at strong edges. Nearly uniform regions cause high peaks in the histogram and large gray values in the final image due to the integration. This limitation can be over comed by restoring the same amount of contrast at each pixel, this is achieved by making bound to the original histogram. This was the main idea behind the CLAHE proposed by Pizer et al. in Pizer et al. in [8].

\subsubsection{Contrast Limited Adaptative Histogram Equalization (CLAHE)}

The contrast Limited Adaptative Histogram Equalization creates images in which the noise is not extremely enhanced, however, an enough contrast enhancement is given for the appearance of structures inside the image. Processing image with CLAHE gives a natural appearance as it provides comparison of area within the image. On the other hand, enhancement with CLAHE may hinder the aptitude of the observer to distinguish some important gray scale levels [9]. Figure 4 shows the result of mammogram enhancement using HE and CLAHE. 


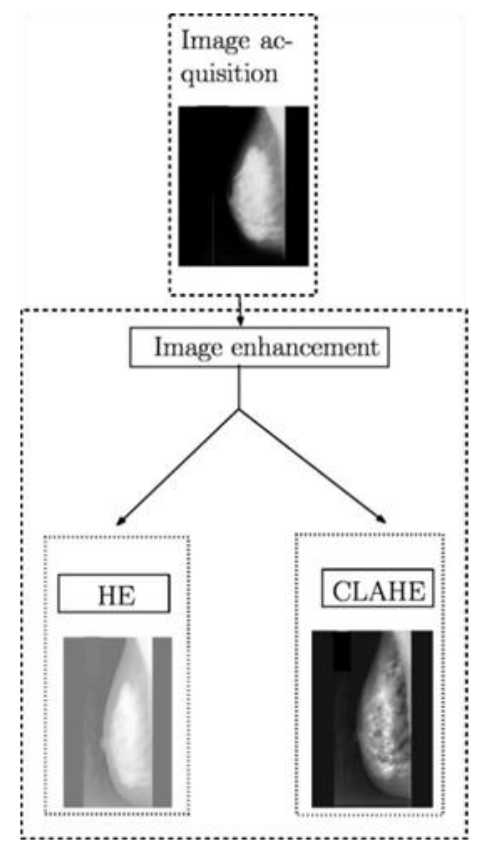

Fig4: Enhancement results on a mammography image using histogram equalization and CLAHE

\section{SHOCK FILTER (SF)}

Shock Filters belongs to the class of morphological image enhancement methods. Most of the current SFs are a modified version of Osher and Rudin's formulation in terms of partial differential equations (PDEs) [10]. The SF was used at the preprocessing stage. It is applied to overcome the problem of noisy fluctuations and enhance edges containing useful information with improvement of the contrast [11] [12]. The idea of shock filtering is explained as follows:

Let $f$ be a continuous image where $f: R^{2} \rightarrow R$ the class of the filtered images $\{u(x, y, t) \mid t \geq 0\}$ may be produced as a result of developing the image $f$ below the process:

$$
\begin{gathered}
u_{t}=-\operatorname{sign}(\Delta u)|\nabla u| \\
u(x, y, 0)=f(x, y)
\end{gathered}
$$

Where subscripts represent partial derivatives, with $\nabla u=\left(u_{x}, u_{y}\right)^{T}$ correspond to the spatial gradient of $u$. This process begins at $t=0$ with $f(x, y)$ corresponding to the original image as shown in condition (2). The progress of the image proceeds as follows:

Suppose that a number of pixels is located in the influence zone of a maximum and its Laplacian function used for edge detection expressed by:

$$
\Delta u=u_{x x}+u_{y y}
$$

The first condition in Eq. (2) demonstrates that the process starts at $t=0$ and the original image corresponds to $f(x, y)$, so Eq .(1) can be written as:

$$
u_{t}=-\operatorname{sign}\left(v_{n n}\right)\left|\nabla_{u}\right|
$$

Such as $n$ corresponds to the direction of the gradient. The edge detector $V_{n n}$ convolved with the Gaussian coefficient $K_{\sigma}$ increase the performance of the $\mathrm{SF}$ and the corresponding equation of SF becomes:

$$
u_{t}=-\operatorname{sign}\left(K_{\sigma}^{*} v_{n n}\right)|\nabla u|
$$

Mammographic images are frequently characterized with low contrast and relatively elevated noise. Enhancement can be achieved by improving contrast, suppressing the background and then the noise, and enhancing shape, boundary and edges. Therefore, we have suggested the Shock Filter to enhance contrast and reduce destroyed areas of the image. The enhancement performance measure of this technique is discussed in the next section.

\section{ENHANCEMENT PERFORMANCE MEASURE AND EXPERIMENTAL RESULTS}

For the evaluation of the proposed approach, we are based on the use of 40 mammograms from the MIAS database [13].We compare the effectiveness of our approach and other enhancement methods using two metrics:

- Contrast measure performance

- The second-derivative measure of enhancement (SDME)

The experimental results will be expressed in the following subsections.

\subsection{Statistical measures of contrast in an image}

To evaluate quantitatively $\theta$ the contrast performance we use the method based on measure of the intensity of the contrast between a pixel and its neighbors over the whole image. Thanks to its efficiency in feature extraction, cooccurrence matrix is the most widely used. It measures the probability of occurrence pairs of pixel values within a certain distance in the image. It is based on the computation of the probability $P(i, j, \delta, \theta)$ which represents the number of times the gray value pixel $i$ appears within a distance $\delta$ to another gray value pixel $j$ and with an orientation $\theta$. The angular orientations $\theta$ which are conventionally used are $0,45,90$ and 135 degrees. With a 256 gray level image, the co-occurrence matrix is $256 * 256$. It records a huge amount of information, difficult to exploit directly; that's why, many authors such as Haralick and al. [14] propose a parameter series of texture from this matrix. Contrast is the most useful and relevant parameter [15], it is expressed as follows: 


$$
C=\sum_{i, j}|i-j|^{2} P(i, j)
$$

The improvement of the mammogram image contrast enhanced, compared to the original and its visual quality of particular regions can be shown in the plotted curves within figure 5. In this figure, we used 40 samples of MIAS database to do the comparison.

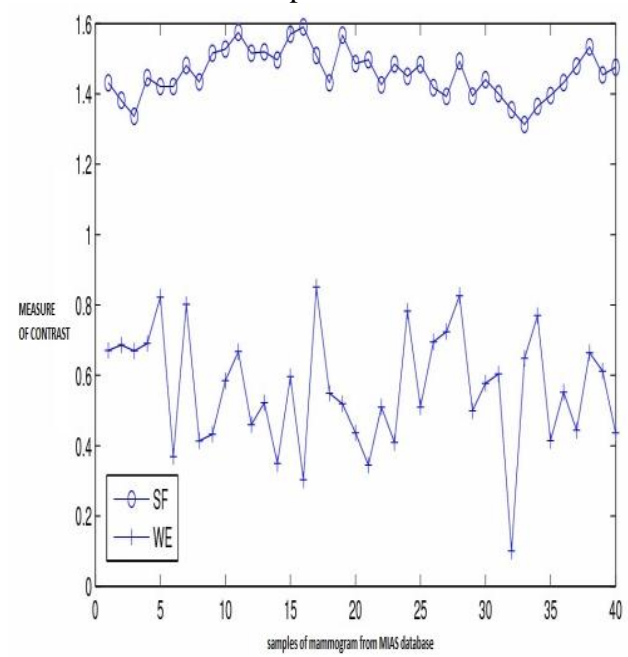

Fig5: Plot of the results of statistical measures of contrast For 40 samples from MIAS database

Table 1 show the result of comparative study of the technique based on SF with NSCT, HE and CLAHE with respect to contrast. The efficiency of the suggested method was verified through the mammogram mdb034 from the MIAS database.

Table 1. Contrast measure results for mammogram enhancement of mdb034

\begin{tabular}{|c|c|}
\hline Method of enhancement & Value of contrast \\
\hline WE & 0.5842 \\
\hline NSCT & 0.5844 \\
\hline HE & 1.1706 \\
\hline CLAHE & 1.4601 \\
\hline SF & 1.5267 \\
\hline
\end{tabular}

So, statistical measures of contrast were mended from 0.5 for the original image and reaches 1.5 with the proposed method. The SF clinical usage renders the elaboration of the image easier in more difficult diagnosis.

\subsection{The second-derivative measure of enhancement (SDME)}

\subsubsection{SDME based measure}

This measure is derived from the concept of the second derivative, it is expressed as:

$S D M E=-\frac{1}{k 1 k 2} \sum_{l=1}^{k_{1}} \sum_{k=1}^{k_{2}} 201 n\left|\frac{I_{\max } ; k, l-2 I_{\text {center } ;, k, l}+I_{\min ; k, l}}{I_{\max ; k, l}+2 I_{\text {center } ;, l}+I_{\min } ; k, l}\right|$
Where the image is divided into ( $k 1 \times k_{2}$ ) blocks with odd size, $I_{\max ; k, l}$ and $I_{\min ; k, l}$ correspond to the maximum and minimum values of pixels in each block whereas $I_{c e n t e r ; k, l}$ is the value of the intensity of the pixel in the center of each block.

\subsubsection{Performance measure}

We measure the performance of the technique based SF by applying SDME. Then, we compare the results of contrast measure using SF with other methods such as NSCT, HE, CLAHE. Figure 6, illustrates an example of comparison.

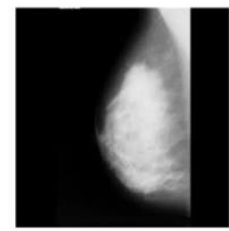

(a) $\mathrm{SDME}=14.808$

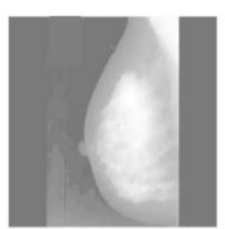

(c) SDME $=13.900$

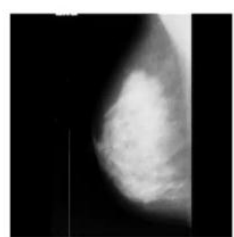

(b) $\mathrm{SDME}=21.667$

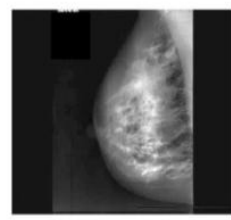

(d) $\mathrm{SDME}=23.880$

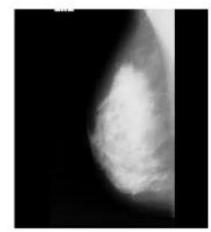

(e) SDME $=26.820$

Fig6: Different Enhancement Techniques. (a) Original image mdb001, (b) image processed by NSCT, (c) image processed by HE, (d) image processed by CLAHE, (b) image processed by $\mathrm{SF}$.

We can notice for fig.6 (a), that the SDME corresponding to the original image is 14.808 , the enhancement by the presented SF improves the visual quality, thus, the SDME is significantly improved to 26.82 and especially in the surrounding of microcalcifications or abnormal regions. In the mammogram presented in fig.6 (d) and fig.6 (e), we see in SF, microcalcifications and abnormal region are overenhanced, that's why it leads to better performance. Also, in fig.6, we are told that Box plots are very useful for comparing multiple data sets, the presented Box plots in fig 7 illustrate the results of comparison between our approach and the recent known method. So, we can appreciate the significant enhancement of the contrast using the Shock Filter. 


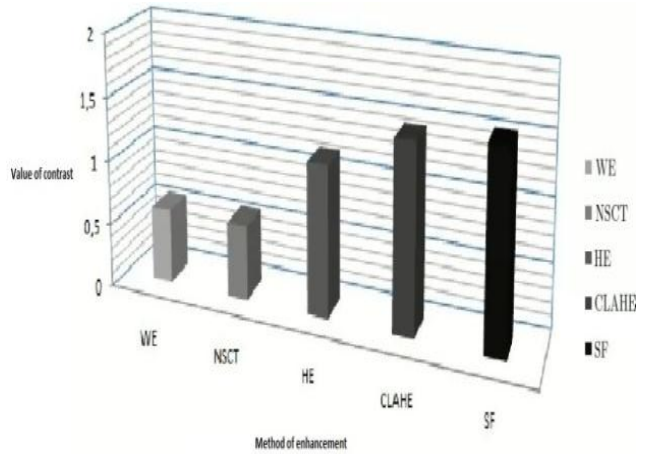

Fig7: Plot of the results of comparison between our approach and the recent known method.

\section{CONCLUSION}

In this paper, an efficient method for enhancement of breast cancer tumor on digital mammogram images has been proposed. It is based on the application of the shock filter. The shock filter has been applied to increase the contrast in mammogram images.

Preliminary experimental results on several images from MIAS database, show that the proposed method yields significantly superior visual quality and contrast compared to other well known methods, the result of the SDME was improved from 14.8 to 26.8 .

Future work should concentrate on mammogram image segmentation, feature extraction and ACR/Birads classification, based on the enhanced mammogram improving the diagnosis accuracy. Further work on this approach is on progress. We are planning to present a fast algorithm providing the desired classification.

\section{REFERENCES}

[1] J. Tang, R. Rangayyan, Y. Yang, I. El Naqa, and J. Xu, "Computer Aided Breast Cancer Detection and Diagnosis Using Mammography: Recent Advance", IEEE Transactions on Information Technology in Biomedicine, 2009.

[2] H. Yoshida, K. Doi., R. Nishikawa, M. Giger and R.Schmidt, "An improved computer-assisted diagnostic scheme using wavelet transform for detecting clustered microcalcifications in digital mammograms", Acad. Radiol, 1996.

[3] Jain, A. K. 1989 Fundamentals of Digital Image Processing. Englewood Cliffs.
[4] E. D. Pisano, E. B. Cole, and B. M. H. e. al., "Image Processing Algorithms for Digital Mammography: A Pictorial Essay.”, Radiographics, 2000.

[5] M. N. Do and M. Vetterli, "The contourlet transform: an efficient directional multiresolution image representation", IEEE Trans Image Process, 2005.

[6] A. L. Da Cunha, J. Zhou, and M. N. Do, "The Nonsubsampled Contourlet Transform: Theory, Design, and Applications", IEEE Trans. Image Process, 2005.

[7] S. M. Pizer, J.B.Zimmerman, and E. Staab, "Adaptive grey level assignment in CT scan display", Journal of Computer Assistant Tomography, 1984.

[8] Pizer, S.M., Amburn, E.P., Austin, J.D., Cromartie, R., Geselowitz, A., Greer, T., Romeny, B.T.H. , and Zimmerman, J.B., 1987. Adaptive Histogram Equalization and Its Variations, Computer Vision, Graphics, and Image Processing.

[9] Rehm, k., and Dallas, W.J., 1989. Artifact Suppression in Digital Chest Radiographs Enhanced with Adaptive Histogram Equalization. presented at SPIE: Medical Imaging III.

[10] S.Osher and L.Rudin, "Feature-oriented image enhancement using shock filters", SIAM Journal on numerical Analysis, 1990.

[11] S.Bettahar, and A.B. Stambouli, "Shock filter coupled to curvature diffusion for image denoising and Sharpening", Journal of image vision and computing, 2008 .

[12] Suganthi, M., and Madheswaran. 2009. Mammogram image enhancement and denoising using shock filters. Proceedings of International Conference on Advanced Communication and Informatics.

[13] Suckling, J., Parker, J., Dance, D.R., Astley, s., Hutt, I., Boggis, C., I.Ricketts, Stamatakis,E., Cerneaz, N. and Kok, S.L. 1994. The Mammographic Image Analysis Society digital mammogram database. Digital Mammography.

[14] R.M. Haralick. "Statistical and structural approachs to texture", Proceedings of the IEEE in Proceedings of the IEEE, 1979.

[15] A. Baraldi and F. Parmiggiani. "An investigation of the textural characteristics associated with gray level occuence matrix statistical parameters", IEEE Transactions on Geoscience and Remote Sensing, 1995. 